\title{
Temperature Dependence of Syringomycin E Channel Functioning in Planar Lipid Bilayers
}

\author{
O. S. Ostroumova ${ }^{a}$, Ph. A. Gurnev ${ }^{b}$, and V. V. Malev ${ }^{a, c}$ \\ ${ }^{a}$ Institute of Cytology, Russian Academy of Sciences, St. Petersburg, Russia; \\ e-mail: ostroumova@mail.cytspb.rssi.ru \\ ${ }^{b}$ National Institute of Child Health and Human Development, Bethesda, Maryland, USA; \\ ${ }^{c}$ St. Petersburg State University, Peterhof, Russia
}

DOI: $10.1134 / \mathrm{S} 1990747809030386$

We have studied conductivity and dwell time of ion channels produced by antifungal lipodepsipeptide syringomycin $\mathrm{E}$ (SRE) in planar lipid bilayers in the temperature range of $10-50^{\circ} \mathrm{C}$. Membranes were formed from either phosphatidylethanolamine or from an equimomolar mixture of phosphatidylserine and phosphatidylethanolamine; membrane bathing solution contained $0.4 \mathrm{M} \mathrm{LiCl}$ or $0.4 \mathrm{M} \mathrm{NaCl}, \mathrm{pH} 6$. We have established that a $10^{\circ} \mathrm{C}$ increase in temperature produces $\sim 1.5$-fold increase in the single-channel conductance and $\sim 3$-fold decrease in the mean dwell time of a single channel. These changes correspond to $\sim 7 \mathrm{kcal} / \mathrm{mol}$ activation energy for ion transport through the SRE channel. This value exceeds the activation energy barrier of the free ion diffusion in water by a factor of two $(\sim 3.3 \mathrm{kcal} / \mathrm{mol})$. Higher value of the activa- tion energy of ion transport in the SRE channel suggests specific interactions between the permeating ions and the channel walls; this suggestion is also supported by our earlier observation of the difference between SRE channel conductance and the conductance of the equivalent water volume. The decrease of the channel dwell time with the increase of temperature may be related to dissociation of the SRE aggregates (6-7 lipodepsipeptide molecules) forming the conducting channel.

The work is partially supported by the Russian Foundation for Basic Research (project no. 06-04-48860), Basic Scientific Schools (project no. 1135.2008.4), and the Program of the Presidium of the Russian Academy of Sciences Molecular and Cell Biology. 